\title{
Analysis of Spatial and Temporal Differentiation Characteristics of Tourism Development Quality of Urban Agglomeration in the central of Yunnan
}

\author{
Anle Liu', ${ }^{1}$, Yanmei Wang ${ }^{1}$, Chengyue Yang ${ }^{2}$, Qingzhong Ming ${ }^{1} 2^{*}$ \\ ${ }^{1}$ Institute of Tourism and Cultural Industry Research, Yunnan University of Finance, Kunming, 650221, \\ China; \\ ${ }^{2}$ School of Tourism and Historical Culture, Liupanshui Normal University, 553004, China. \\ * Corresponding author, AnLe Liu and YanMei Wang contributed equally to this paper
}

Keywords: Tourism development quality; comprehensive measure; space-time differentiation; urban agglomeration of central Yunnan

\begin{abstract}
In order to explore the characteristics of tourism development stage and its spatial differentiation characteristics in the urban agglomerations of central Yunnan(UACY), the standard deviation, weighted coefficient of variation, Herfindahl index, entropy method and AcrGIS10.0 software were used to analyze characteristics of tourism development in the UACY, the study found that: Since 1990, the tourism development of the UACY has experienced four stages: start-up, rapid development, volatility, and transformation. The quality of tourism development shows the characteristics of "aggregation-distribution" in time-space evolution; The quality of county-level tourism development in the UACY is closely related to its tourism economic strength, tourism resources and tourism service facilities; Among the UACY, the developed tourism region are not obvious beneficial to the development of surrounding region. The quality of tourism development presents a regular pattern of gradually decreasing from the south to the periphery of the urban agglomeration. The findings in this paper can provide a realistic reference for the holistic tourism destination and regional coordinated development of the UACY.
\end{abstract}

\section{Introduction}

Correctly understanding the quality of regional tourism development is an important reference for formulating regional tourism development policies and strategies. 'The Outline of Tourism Quality Development (2013-2020)' clarifies that perfecting the tourism quality evaluation system is one of the five key grasps for consolidating the foundation of tourism quality development. Therefore, under the new normal of tourism industry development, especially the all-for-one tourism construction and reform of supply-side of tourism industry, the researches on tourism development quality have important practical value for layout and optimization of the regional tourism industry.

By reviewing the literature of tourism development quality at the present stage, it is found that the existing research results mainly introduce and construct the tourism development quality evaluation index system and conduct empirical research[1-5] from the field they focused for long-term. The relevant research results are mainly concentrated on the construction of quality evaluation index system of regional tourism development and empirical research about collaborative relationships between tourism development quality and related industry [6-8]. These empirical researches have laid a solid foundation for the systematic evaluation of the tourism development quality in this paper. However, in terms of research area and scale, the macro-regional and economically developed regions are mainly focused instead of the western region. In particular, researches on tourism development of urban agglomerations in the western of China need to be sorted out.

UACY is the core tourist area of Yunnan Provincial tourism development overall layout which targeted as "One Heart, Six Districts and Seven Corridors" and international leisure and holiday tourist destinations for Southeast Asia and South Asia. It is an important growth pole for tourism industry economic development and also plays a pivotal role in future tourism development of Yunnan Province, especially in terms of "start a new undertaking of Yunnan tourism". Therefore, exploring the 
characteristics of tourism development stage and spatial differentiation can provide a realistic reference for all-for-one tourism construction and regional coordinated development of UACY.

\section{Overview of the Study Area}

The urban agglomeration in central Yunnan is a place at latitude $24^{\circ} 00^{\prime}$ to $26^{\circ} 30^{\prime}$ North, longitude $101^{\circ} 00^{\prime}$ to $104^{\circ} 30^{\prime}$ East, it is located in the core area of the Yunnan Plateau, shares border with Guizhou Province in the east, Sichuan Province in the north, and is connected to Dali, Pu'er, Honghe and Wenshan in the west and south respectively. It spans approximately $960,000 \mathrm{~km}^{2}$, which accounts for $24 \%$ of the whole province. The annual average temperature is from 15 to $18^{\circ} \mathrm{C}$, and has a generally mild climate with pleasant and fair weather. The tourism resources here are very rich and concentrated, formed a peculiar natural tourism resource, rich and colorful national cultural tourism resources. By the complementing and blending of these two points, the UACY has an incomparable superiority to the market.

\section{Methodology}

\subsection{Research methods}

Measurement method of tourism development quality: The fuzzy comprehensive evaluation method can be used to objectively and realistically evaluate the comprehensive strength of tourism development in UACY. The specific calculation process is as follows:

$$
T=\sum_{i=1}^{i} u_{i t} t_{i}
$$

$T$ is the comprehensive evaluation value of tourism development quality; $t_{i}$ is the standardized value of tourism development level, $u_{i}$ is the index weight of tourism development level, the weight of index is calculated by entropy method [9].

\subsection{Tourism Development Quality Evaluation Index System}

Tourism is a complex and chaotic regional system consisting of a number of factors that all complex systems interacts and subsystems intermingles with each other. This paper follows the principles of hierarchy, simplicity and guidance. Based on the research results of Zhu mei, the paper combines with the actual development of the tourism industry in the UACY, through consulting experts, 15 indicators are selected to construct the county tourism development quality evaluation index system[10] from the tourism economy (TE), tourism resource competitiveness (TRC), tourism reception service foundation (TRF) and tourism environment background (TEB).

Table 1 Evaluation Index System of Tourism Development Quality in the UACY

\begin{tabular}{cclc}
\hline Target layer & Index category & \multicolumn{1}{c}{ Index layer } & Weight \\
\hline & & Foreign Exchange Earnings $C_{1}$ & 0.0532 \\
& & Domestic tourism revenue $C_{2}$ & 0.0695 \\
& $T E$ & Gross tourism revenue as a share of GDP $C_{3}$ & 0.0323 \\
& $(0.3207)$ & Inbound tourist $C_{4}$ & 0.0762 \\
Tourism & & Domestic tourist $C_{5}$ & 0.0511 \\
Development & & Tourism staff ratio $C_{6}$ & 0.0384 \\
Quality $(T Q)$ & \multirow{2}{*}{$T R C$} & Tourism resource abundance ${ }^{*} C_{7}$ & 0.0911 \\
& $(0.2774)$ & Tourism resource taste* 8 & 0.1023 \\
& & Main Scenic Attraction $($ spot) Location Dominance*C9 & 0.0840 \\
\cline { 2 - 4 } & \multirow{2}{*}{$T R F$} & Number of travel agencies $C_{10}$ & 0.0925 \\
& $(0.2628)$ & Number of star hotels $C_{11}$ & 0.0980 \\
& & Annual reception of scenic attraction more than $10^{4} \mathrm{C} 12$ & 0.0713 \\
\hline
\end{tabular}

(1) The definition of Urban Agglomeration of central Yunnan in this paper is according to the public announcement of "Central Yunnan Urban Agglomeration Planning (2009-2030)". 


\begin{tabular}{clc}
\hline \multicolumn{3}{c}{ Cont. to Table 1 } \\
\hline \multirow{2}{*}{$T E B$} & The number of days air quality above good $C_{13}$ & 0.0351 \\
$(0.1391)$ & Per capita public green area $C_{14}$ & 0.0506 \\
& July temperature suitability* $C_{15}$ & 0.0534 \\
\hline
\end{tabular}

In this paper, the entropy method is used to obtain the value of the evaluation factor weight, which can effectively avoid the deviation problem caused by the subjectivity of expert scoring. The actual data of the indicators are listed in the article is obtained from the Statistical Yearbook of the UACY in 2016, the Statistical Communiqué of the National Economic and Social Development, and the official website of the Tourism Bureau, the Environmental Protection Agency and related units. Among them, C7: this value is calculated from the total numbers of world natural and cultural heritage, national historical and cultural cities, nationally important scenic and historic interest area, national nature reserves, national forest parks, and tourist attractions above $3 \mathrm{~A}$ in each county unit (multiple titles in the same scenic spot are not repeated in calculations) [11]; C8: Through assigning value to different levels of tourism resources in each county unit, the specific assignment is: World natural and cultural heritages, national 5A attractions all count 10 points, national 4A attractions and national parks of China count 5 points, the national nature reserves count 3 points, the national forest parks count 2 points, the multiple titles of the same scenic attraction in the county unit is calculated according to the highest title, and the final score of each item in the county unit is added as the score of tourism resources taste[11]. C9: The shortest average travel time to Kunming, Qujing, Yuxi and Chuxiong is calculated as the location advantage value of scenic attraction of this county by selecting the top three scenic attractions for tourism reception. C15: According to the following methods, it is generally considered that $18-22^{\circ} \mathrm{C}$ outdoor in summer is the best suitable temperature for human apparent temperature, and the UACY is an important summer tourism destination, so the average temperature in July of the county unit is collected. By comparing with the best suitable temperature, the absolute value of the temperature difference less than $1{ }^{\circ} \mathrm{C}$ counts 5 points, while 4 points is the absolute value between $1-2^{\circ} \mathrm{C}, 2$ points is between $2-4^{\circ} \mathrm{C}, 1$ point is between the $4-5^{\circ} \mathrm{C}, 0$ point is the value higher than $5^{\circ} \mathrm{C}$, the final score of each county unit is the annual temperature suitability score.

\section{Sequential Characteristic of Tourism Development in UACY}

\subsection{Analysis on the characteristics of tourism development stage}

UACY is the first open tourism destination and tourism development core in Yunnan Province. Its tourism development process is similar to the overall development process of Yunnan Province. This paper mainly focuses on the development status of tourism in the historical period of UACY. According to the development of UACY, the tourism development process is divided into four stages (Fig. 1):

The initial stage (1990-1995): In 1978, the official establishment of the Yunnan Tourism Bureau marked the start of development of Yunnan tourism. However, in the following 10 years, the tourism sector was positioned as a "non-productive" department. Until 1988, the work report of the seventh national people's congress of Yunnan province confirmed the major industry status of tourism. At this point, the tourism development of the UACY really started. In 1993, Yunnan province issued the "Opinions on Developing Tourism Industry Vigorously", which created a new situation in the tourism development. By 1995, the number of tourist receptions in the case area reached 9.1492 million, the annual average growth rate reached $80.2 \%$; the tourism revenue increased to 2.219 billion RMB, the average annual growth achieved $150 \%$. Although it had achieved great development, this period is still in its infancy.

Rapid development stage (1996-2000): In 1996, the Yunnan Provincial Party Committee and the Provincial Government issued the "Decision on Accelerating the Construction of Four Pillar Industries". The UACY successively established tourism administrative institutions. In this time, the concept of the UACY was proposed and written into the "Kunming, Qujing, Yuxi, Chuxiong Urban Agglomeration Plan ", which integrated tourism into the overall strategy of economic and social development, this contributed to the rapid development of the tourism industry of UACY. From 1996 to 2000, the number of tourist 
receptions in the case area increased from 17, 061, 540 to $17,990,800$, the average annual increase was $33.4 \%$; tourism revenue increased from 4.133 billion RMB to 11.736 billion RMB, average growth per annum was $56.7 \%$, tourism industry had been consolidated and developed as the pillar industries in Yunnan urban agglomeration.

The advanced in volatility stage (2001-2005): At this stage, due to the impact of SARS in 2003, tourism reception and tourism income fluctuated, but under the support of strong policies which was "General planning of tourism development in Yunnan Province(2001-2020)", "Yunnan Tourism Development Multiplication Plan”, “Eleven-Five' Tourism development plan in Yunnan Province”, from 2001 to 2005, the number of tourist receptions in the UACY increased from 26.88 million to 32.544 million, the annual growth was $24.2 \%$; The tourism income increased from 17.837 billion RMB to 15.88 billion $\mathrm{RMB}$, average growth per annum was $22.3 \%$. It was the rapid growth of the tourism industry in the UACY, which indicates that the tourism industry development in this area is gradually mature.

Transformation and upgrading stage (2006-present): Since 2006, The UACY has actively promoted the tourism industry from sightseeing type to leisure type and built China international leisure tourism area under the policy of "second undertaking" of Yunnan tourism industry and the development strategies of "Drive development by full launch and implementation of major projects". Also, it has actively cultivated and built "Kunming Yuxi culture and leisure tourism belt" and "Kunming Chuxiong cultural tourism belt", and has improved the leisure tourism area surround the Dianchi lake, Jiaozi snow mountain eco-tourism area, Fuxian lake plateau water town leisure resort, mountain landscape leisure tourist area including Xiu mountain-E mountain, Chuxiong "Three A and One I "(2) cultural leisure tourism area, Xundian- Malong- source of pearl river health and leisure tourism area and other six leisure tourism areas[12]. Until 2016, the total tourism revenue of the four cities and municipalities in the UACY has reached 155.761 billion $\mathrm{RMB}$, accounting for $35.9 \%$ of the provincial total tourism revenue, and the number of tourists was 17.09325 million, accounting for $39.7 \%$ of the provincial gross tourism revenue.

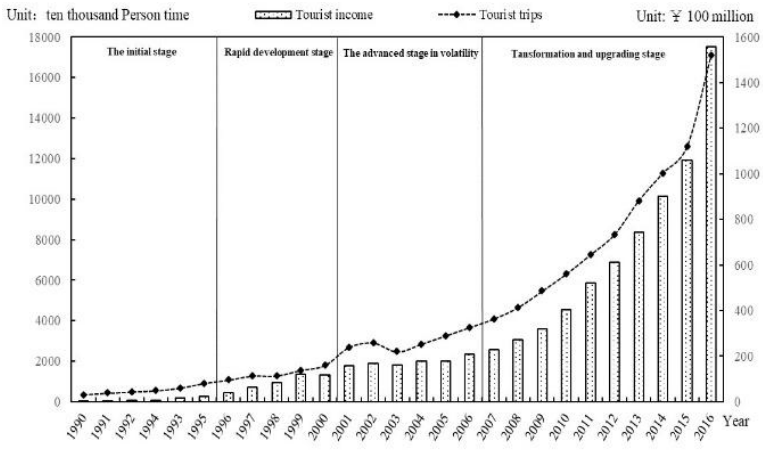

Fig.1 Annual changes and stage division of tourism development in the UACY

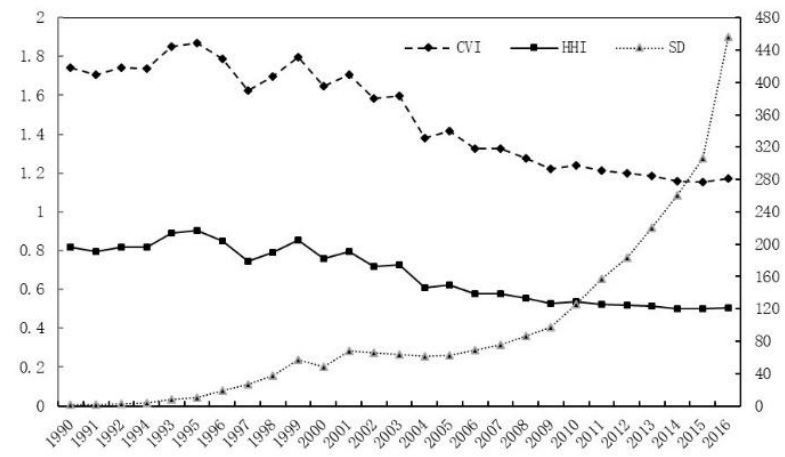

Fig.2 Time evolution of spatial differences in tourism development quality among the UACY

\subsection{Timing changing characteristics of spatial differences in tourism development}

According to the standard deviation $(S D)$, weighted coefficient of variation $(C V I)$ and Herfindahl index $(\mathrm{H})$ of Yunnan's tourism economy are calculated, which quantitatively reflect the tourism spatial differences characteristics of tourism development in the UACY(Fig. 2) .

Through analysis, it is found that from 1990 to 2016, the standard deviation of tourism development quality in the UACY is continuously rising; this indicates that the absolute difference in space of tourism development quality in the counties and districts of the UACY is expanding. The inter-annual variation from the weighted coefficient of variation can be divided into three stages: From 1990 to 1995 is a slow rising phase, the weighted coefficient of variation rises from 1.741 to 1.869 ; the volatility decline stage is from 1996 to 2005, the weighted coefficient of variation decreases from 1.786 to 1.324 and is steadily decreasing after 2006.It is consistent with the annual change of Herfindahl index, this means the relative

(2) "Three A and One I" represents the Yi national minority culture, Yuanmou Man, Paleontology, Ancient Cultural in Chuxiong. 
differences in the UACY shows expansion trend firstly, fluctuation reduction next and continuous reduction trend finally. The absolute difference in the quality of tourism development among the UACY has been expanding from 1990 to 2016, these indicates that the characteristics of spatial pattern evolution of tourism development spatial pattern experienced "aggregation-distribution" trend.

\section{Spatial Differentiation Characteristics of Tourism Development Quality in the UACY}

\subsection{Measurement results of tourism development quality in case area}

The tourism development quality sub-index and the tourism comprehensive development quality index of 42 county unit of UACY are calculated by the above research methods. The results are shown in Table 2 .

In tourism development quality ranking, the counties' ranks of tourism economic, tourism resource competitiveness, tourism reception service base and tourism comprehensive development quality in the UACY shows significant consistency trend, which indicates a closely relationship between tourism development quality and tourism economic strength, tourism resources and tourist service facilities. Further analysis believes that tourism economic strength, tourism resources and tourism service facilities determine the quality of tourism development in this area to some extent. The ranks between tourism environment background and tourism comprehensive development quality are quite different, and the author adjusts the ranks of tourism development quality of county units according to quality of the tourism environment background. As the whole, the excellent background of tourism environment can improve the ranks of tourism development quality in the county, on the contrast, the quality is poor. Further analysis believes that although the tourism environment background is not the determining factor of county tourism development quality, it plays a vital role in county tourism development. Therefore, in the process of region tourism development, we should focus on environmental protection.

Table 2. Results and ranking of tourism development quality in the UACY

\begin{tabular}{ccccccc|ccccccc}
\hline County & $T E$ & $T R C$ & $T R F$ & $T E B$ & $T Q$ & Ranks & County & $T E$ & $T R C$ & $T R F$ & $T E B$ & $T Q$ & Ranks \\
\hline Guandu & 0.29 & 0.20 & 0.26 & 0.13 & 0.87 & 1 & Wuyi & 0.06 & 0.10 & 0.04 & 0.05 & 0.25 & 22 \\
Xishan & 0.26 & 0.27 & 0.25 & 0.06 & 0.84 & 2 & Huaning & 0.03 & 0.06 & 0.02 & 0.14 & 0.25 & 23 \\
Panlong & 0.26 & 0.20 & 0.20 & 0.06 & 0.72 & 3 & Shuangbai & 0.03 & 0.08 & 0.01 & 0.13 & 0.24 & 24 \\
Wuhua & 0.23 & 0.13 & 0.20 & 0.09 & 0.65 & 4 & Yuanmou & 0.11 & 0.10 & 0.04 & 0.00 & 0.24 & 25 \\
Chuxiong & 0.10 & 0.24 & 0.14 & 0.14 & 0.62 & 5 & Jinning & 0.03 & 0.09 & 0.03 & 0.09 & 0.24 & 26 \\
Shilin & 0.08 & 0.18 & 0.10 & 0.14 & 0.50 & 6 & Malong & 0.04 & 0.08 & 0.03 & 0.09 & 0.23 & 27 \\
Hongta & 0.11 & 0.19 & 0.11 & 0.09 & 0.50 & 7 & Xundian & 0.02 & 0.05 & 0.04 & 0.13 & 0.23 & 28 \\
Jiangchuan & 0.08 & 0.13 & 0.05 & 0.14 & 0.40 & 8 & Mouding & 0.02 & 0.06 & 0.02 & 0.13 & 0.22 & 29 \\
Xinping & 0.04 & 0.15 & 0.05 & 0.14 & 0.38 & 9 & Fuming & 0.04 & 0.07 & 0.02 & 0.09 & 0.21 & 30 \\
Chengjiang & 0.09 & 0.10 & 0.03 & 0.14 & 0.37 & 10 & Luquan & 0.00 & 0.08 & 0.03 & 0.09 & 0.21 & 31 \\
Qilin & 0.05 & 0.11 & 0.10 & 0.09 & 0.35 & 11 & Huize & 0.04 & 0.07 & 0.01 & 0.09 & 0.20 & 32 \\
Yiliang & 0.03 & 0.11 & 0.06 & 0.14 & 0.34 & 12 & Chenggong & 0.01 & 0.09 & 0.03 & 0.06 & 0.20 & 33 \\
Lufeng & 0.07 & 0.11 & 0.02 & 0.13 & 0.32 & 13 & Shizong & 0.04 & 0.08 & 0.02 & 0.05 & 0.19 & 34 \\
Tonghai & 0.06 & 0.10 & 0.01 & 0.14 & 0.31 & 14 & Songming & 0.01 & 0.06 & 0.03 & 0.09 & 0.19 & 35 \\
Ershan & 0.05 & 0.09 & 0.02 & 0.14 & 0.30 & 15 & Yaoan & 0.02 & 0.04 & 0.00 & 0.13 & 0.18 & 36 \\
Luoping & 0.06 & 0.10 & 0.03 & 0.09 & 0.28 & 16 & Fuyuan & 0.02 & 0.08 & 0.03 & 0.05 & 0.18 & 37 \\
Nanhua & 0.06 & 0.10 & 0.02 & 0.09 & 0.26 & 17 & Xuanwei & 0.04 & 0.03 & 0.04 & 0.07 & 0.17 & 38 \\
Anning & 0.08 & 0.11 & 0.02 & 0.06 & 0.26 & 18 & Dayao & 0.02 & 0.05 & 0.01 & 0.09 & 0.17 & 39 \\
Luliang & 0.02 & 0.13 & 0.02 & 0.09 & 0.26 & 19 & Yuanjiang & 0.05 & 0.05 & 0.04 & 0.03 & 0.17 & 40 \\
Zhanyi & 0.02 & 0.15 & 0.03 & 0.05 & 0.25 & 20 & Dongchuan & 0.01 & 0.03 & 0.03 & 0.09 & 0.17 & 41 \\
Yimen & 0.04 & 0.06 & 0.01 & 0.14 & 0.25 & 21 & Yongren & 0.05 & 0.00 & 0.00 & 0.11 & 0.16 & 42 \\
\hline
\end{tabular}




\subsection{Characteristics of the spatial pattern of tourism development quality in UACY}

According to the distribution structure of tourism development quality index of Yunnan urban agglomeration, the AcrGIS10.1 are used to divide this area into four regions (Fig. 3).

The tourism development of the UACY is polarized in the distribution of county tourism development quality index. Among them, the highest comprehensive quality of tourism development is Guandu (0.872), and the lowest is Yongren (0.162). The difference between these two is over 7 times. Chenggong $(0.196)$, Songming (0.189), Fumin (0.214) and other counties' tourism development quality are all underdeveloped areas and are quite different with the surrounding developed region including Guandu (0.872), Xishan (0.840), Panlong (0.722) and Wuhua (0.647), so the tourism developed region are not obvious contribute to the area around.

In terms of spatial pattern, the quality of tourism development in the UACY is quite different. The quality of tourism development in central cities is relatively high, and the overall characteristic of the urban agglomerations is gradually decreasing from the central south to the periphery of the case area. The traditional main city of Kunming (including Guandu, Xishan, Panlong \& Wuhua) becomes the center of this area in tourism development, while the northern and southern marginal counties becomes the "lowland". Chenggong-Songming- Luquan and other districts connects these centers and the northern "lowland", forming a "valley belt" which is from the center to the north of the urban cluster in the UACY.

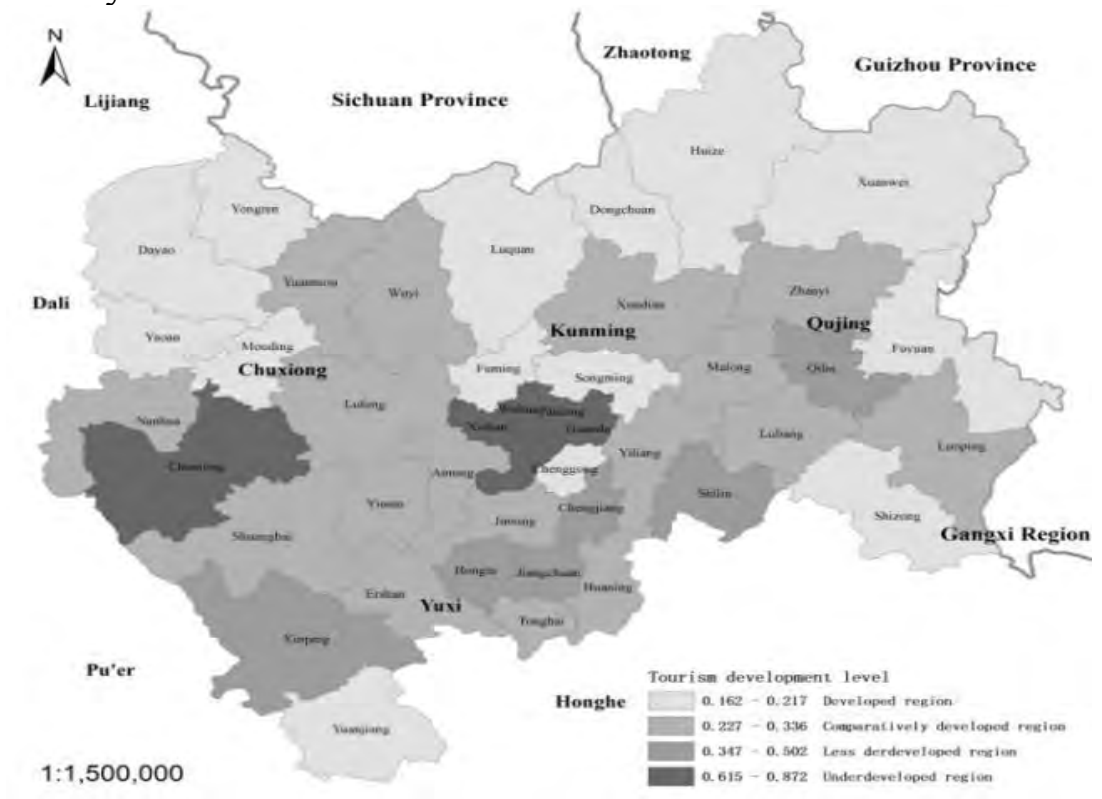

Fig.3 The spatial pattern of tourism development quality in the UACY

Analysis on reasons: economy of traditional main city of Kunming which is the core of the case area with combination of better tourism resources and tourism infrastructure, is relatively developed, so does the tourism development. Northern and marginal counties of urban agglomerations due to economic development level and the restrictions on geographical conditions, failed to effectively transform the tourism resources and tourism environment background advantages into product advantages, therefore, hindered the overall tourism strength development of these county unit. "valley belt" has superior geographical location conditions, the tourism development resource abundance is good, but it is difficult to form a tourist loop. Because of the lower taste of tourism resources and lacks of tourism transportation in the northern region, the slowly tourism development is resulted.

\section{Conclusions and Discussions}

Based on the analysis above, the main conclusions are as below:

First, since 1990, the tourism development process of the urban agglomerations in central Yunnan can be divided into four stages: start-up, rapid development, advanced in volatility and transformation and 
upgrading. The absolute difference in the quality of tourism development in the case area has been expanding, and the relative differences have expanding first and fluctuating then, shrinking and continuously decreasing trend. The quality of tourism development shows the characteristics of temporal and spatial evolution trajectory of "aggregation-distribution" overall.

Secondly, there is a significant consistency between the tourism economic pattern, the tourism resource competitiveness pattern, the tourism reception service basic pattern and the tourism comprehensive development quality pattern in the UACY, this indicates the quality of tourism development of the county-level in central Yunnan urban agglomeration are closely related with its tourism economic strength, tourism resources and tourism service facilities.

Third, the tourism development of the UACY is highly polarized; the quality of central cities is relatively high. The developed tourism regions are not obviously contributed to tourism of surrounding area. The overall tourism development shows regular feature that gradually declines from the south to the periphery of the urban agglomeration and forms a "valley belt" connected by districts and counties such as Chenggong-Songming-Fumin-Luquan.

Fourth, due to the lack of data in the research process, the spatial pattern difference is only explored by the 2015 data, and there is no comparative study of the diachronic line. The changing trend of the regional spatial pattern difference needs to be further explored.

\section{Acknowledgment}

This paper is supported by National Natural Science Foundation of China(41671147); Supported of Innovation Group by Educational Commission of Guizhou Province of China (QianJianHe KY Zi [2016]056NO.); Supported of Tourism Industry Research base of Yunnan Province of China(JD2017ZD02); Supported of Technology Innovation Team of Liupanshui Normal University (LPSSYKJTD201604).

\section{References}

[1]. WEI Jie, LI Meng and REN Bao-ping. "The Temporal Characteristics and Regional Difference of the Quality of Tourism Development in China". Journal of Business Economics, 2016, vol.10, pp. 78-87.

[2]. ZHANG Ai-ping, ZHONG Lin-sheng and XU Yong, etc. "Characteristics and Spatial Difference of Provincial Tourism Development Quality in China”. Scientia Geographica Sinica, 2015, vol.35(03), pp.283-292.

[3]. CHEN Xiu-qiong, HUANG Fu-cai. "A Research on Quantitative Evaluation of Tourism Growth Quality in China”. Tourism Tribune, 2006, vol.21(09), pp.59-63.

[4]. SONG Chang-hai. "Evaluation index system and index compilation method of tourism development quality”. Statistics \& Decision, 2016, vol.05, pp.39-42

[5]. SHENG Yan-chao. "On the Measure and Evaluation of Regional Tourism Industry Development". Journal of Hunan University of Commerce, 2016, vol.23(01), pp. 58-66.

[6]. LIU An-le, MING Qing-zhong and YANG Cheng-yue, etc. "Analysis of Coordination Degree and Spatial Pattern between Tourism Industry and Urban Development". Journal of Yunnan Normal University (Natural Sciences Edition), 2015, vol.28(03), pp.56-61.

[7]. YU Jie. "Evalution and Optimization of the Coordinative Degree between Tourism Industry and Regional Economy in Shandong Province". China Population, Resources and Environment, 2014, vol.24 (04), pp. 163-168.

[8]. WANG Zhi-min. "Research on the synergetic degree between regional tourism industry and scientific innovation in Jiangsu Province”. World Regional Studies, 2016, vol.25(06), pp.158-165. 
[9]. WANG Feng. "A Research on the frontier area traffic and tourism spatial structure coupling and related mechanism-A Case of Yunnan”. East China Normal University, 2014.

[10]. ZHU Mei, WEI Xiang-dong. "Construction and Application of Index System on Measuring International Tourist City”. Economic Geography, 2011, vol.31(1), pp.170-176

[11]. ZHANG Hai-xia. "Study on tourism regulation of national parks of China". East China Normal University, 2010.

[12]. LUO Ming-yi, XU Nan-yuan, XU Hong, etc. "Research on the cultivation of leisure tourism destination: Theory, demonstration and case". Beijing: Science Press, 2013. 